\title{
Analysis on the Innovative Business Model of Entrepreneurial Short Video Platform in China
}

\author{
Ling Jiang ${ }^{1} \&$ Haiyue Zhang ${ }^{1}$ \\ ${ }^{1}$ College of Communication and Art Design, University of Shanghai for Science and Technology, Shanghai, China \\ Correspondence: Ling Jiang, Ph.D. \& Lecturer, College of Communication and Art Design, University of Shanghai \\ for Science and Technology, 4-504 Apartment No.558 tian bao Road, Hong Kou, Shanghai, China.
}

Received: November 23, 2019

Accepted: December 3, 2019 Online Published: January 31, 2020

doi:10.5430/ijba.v11n1p22

URL: https://doi.org/10.5430/ijba.v11n1p22

\begin{abstract}
In many short video platforms around the world using MCN mode as the main expansion of the video distribution, ranging from the Tik Tok, Youtube to other, "Yitiao" is completely with the PGC mode. The production content, the positioning of the audience as the middle class, the launching of original short videos, and the unique aesthetic content and accurate audience positioning, have revolutionalized the short video industry. With the use of innovative business model, "Yitiao" brings the content and interconnected new media features into the commercial e-commerce field, and combines innovation with offline cultural output. The problems arising from this, such as the lack of strong technical support, and the commercialized and countered core content are not negligible, and they may affect the vitality of monetizing content e-commerce.
\end{abstract}

Keywords: short video, e-commerce, innovative business model

\section{Introduction}

With the advent of the era of mobile Internet, short video with short duration and light weight has gradually become one of the main forms of media communication, and the core content of self-media entrepreneurship has also changed from literalization to videoization. A series of short video self-media entrepreneurship, such as "Ergeng", "Yitiao", "Pear Video", "Tik Tok" and "Kuaishou", has become more and more mature. Against the background of mature development, how to carry out innovative monetization has become the core issue of the industry.

From the realization of UGC content production to the payment of PGC knowledge field, commercial content designated for advertising realization has always been the core of short video entrepreneurial monetization. According to the 2017 mobile Internet video information streaming advertising value research report, China's video information streaming advertising revenue increased by about $20 \%$ year-on-year to about 9.58 billion yuan, and the advertising revenue of short video platforms such as "Tik Tok" and "Kuaishou" has exceeded 1 billion yuan.

However, after advertisement monetization has become the main body of monetization in the field of short video, "Yitiao" platform has reduced the share of traditional advertisement monetization, instead focusing on the "content e-commerce" monetization model, and has opened three "e-commerce offline experience life pavilions". According to the data report, in the beginning of the launch of "Yitiao" app platform in 2016, the monthly revenue has exceeded 10 million yuan, and reached 50 million yuan in January 2017. Now, the monthly sales revenue of commodities has already exceeded the billion yuan mark, completing multiple rounds of financing, and the market valuae has exceeded 2 billion yuan.

"Yitiao" is unique in the cashing mode of the self-media industry, differentiating itself from the short video self-media platform of content entrepreneurship to the "content e-commerce". How to attract the audience, how to integrate the e-commerce platform into PUGC model, and how to monetize the ready-made function of innovation has its unique value and innovation significance.

\section{The Necessity of "Yitiao" App Based on Innovative Business Model}

The first strand of thought, marked by the use of the term "industry", demonstrates a continuity with the cultural industry thinking - namely, thinking about the arts and media in economic terms. At present, the business mode in the short video industry is still dominated by advertising. In addition to the MCN short video platform based on "Tik Tok" and "Youtobu", the PGC content is the core of the entrepreneurial self-media. The platform tends to choose 
"soft and wide" content that matches its own brand image. After the platform "Yitiao" reached the peak of the monetization of advertising, the APP launched by the transformation immediately passed the form of e-commerce, which not only strengthened the core of its brand, but also got rid of the peak limitation brought by the innovative business model of traditional advertising. From advertising to e-commerce, the successful transformation of the "Yitiao" business model relies mainly on the following points:

\subsection{Mainly "Selected" to Continue the Brand Style}

At present, the monetization model in the short video industry is still dominated by advertisements. In addition to UGC short video platforms, such as "Tik Tok", "Kuaishou" and "Meipai", which are mainly based on SMD advertising, more platforms with PGC content as the core tend to choose "soft-ads", "Ergeng", "Instant Video" and other short video platforms that meet their own brand image through shooting brand customized advertisements. At the same time of monetization of "soft-ads" and maintenance of its own brand core value, but there are peaks and peaks in both patch advertising and "soft-ads". Once the advertising changes to a certain degree of commercial interests, there will be a state of stagnation at the highest point, and there is no space to rise. After reaching the peak of advertising cashing, the app transformed and launched immediately not only strengthens its brand core, but also gets rid of the peak limitations brought by advertising cashing through e-commerce.

Since "Yitiao" has established a clear product value core in the early stage and carried out careful marketing in the form of multi-platform short video, it has become a PGC in the field of life aesthetics from a self-media start-up UGC, and has accumulated a large number of fans in this field.

Therefore, on the one hand, "Yitiao" brings this core product value into the transformation and monetization of its own e-commerce, which is more than an integrated shopping platform, selling products with life aesthetics and quality after "selection" and "strict selection" on the platform, echoing the brand core values. Its e-commerce platform includes more than 1000 brands, more than 10000 good products, covering more than 20 different fields.

On the other hand, through its own electricity supplier APP and its own micro-blog public number and other media accounts, "Yitiao" echoes between the two sides. In addition to further embedded the brand core into the audience, the selling of third party products in this kind of electronic business model is completely free and vulnerable to personal platform control. And compared with advertising form, there is no commercial pole and peak with little limitations, thus small profits can also be maximized.

\subsection{Using D2C Business Model, Accurate Positioning From the Followers to the Consumers}

"Yitiao" innovative business model is called D2C mode in the Internet industry, which refers to the product. After designing and producing the goods, the designer directly faces the consumers through a platform, and it is easy to for buyers and sellers to trade in the business model.

Due to the vertical fan audience accumulated in the "Yitiao" period, the middle class of the consumer group who positioned the quality of life believe that they face many niche designer products. The "Yitiao" APP helps these small and humanistic designer products to directly target audience. For example, a series of original products such as the silver pot filled with traditional Chinese aesthetics was introduced and auctioned by "Yitiao" APP, the price raised to over 10,000 yuan.

Once "Yitiao" accurately positioned its own content audience, and over time, with the establishment and deepening of platform reputation and personal design, it attracted a large number of original designers. In the content-based business model, "Yitiao" app more accurately positioned its own consumers, generated two-way profits, connected and communicated through its own platform, and guaranteed consumers and designers.

Compared with traditional e-commerce such as "Taobao", "Jingdong", "Amazon", which focuses on the commercial output of consumer shopping products, the e-commerce mode adopted by "Yitiao" is known as the D2C model in the Internet industry. It refers to the business model that the designer of the product designs and produces the product, directly faces the consumer through a certain platform, providing convenience for buyer and the seller to trade with each other.

Because the vertical fan audience accumulated in the early stage of "Yitiao" is located in the middle class of quality of life, which is the consumer group for many small designers' products, "Yitiao" app gives these small designers' products with humanistic feelings of a direct audience orientation. For example, "Yitiao" app launched a series of original designer products. Consumers no longer need to screen and identify products that meet their own life orientation, and designers don't need to worry about the dwarfing of products due to the stereotype of the platform. 


\section{The "Yitiao" App Business Model With the Innovative Path}

After determining the necessity and business model of e-commerce monetization, "Yitiao"APP has made its own two innovations in the process of monetizing, namely, how to integrate the core value of its content with commercial monetization and how to connect online and offline experience, thus creating a secure and converged platform, .

\subsection{Original Video: "Content-Based" E-Commerce, Get Rid of Third-Party Platform Restrictions}

The original "Yitiao" video is the core foundation for the initial attraction of the audience and the new media platform. As of September 2017, among more than 1 million subscribers of "Yitiao"m more than 30 million registered and used "Yitiao" APP, with an average monthly sales of 100 million yuan.

Looking at various forms of short video cashing in the industry, for short video serving content, content cashing is a hot word in the short video industry. At present, the short video platform appearing in the market mostly adopts the model of content cashing. However, this cashing model usually needs the support and grafting of the third platform.

Taking "Ergeng" as an example, its fusion media platform with multiple platforms, including micro-blog WeChat public headline and so on, has realized a large part of the cashing with the help of the third party platform and micro-blog accounts. "Ergeng" has registered more than 30 micro-blog sub accounts after opening the "Geng-city" sub-station series, resulting in a large portion of fans streaming. So far, "Ergeng" is still not separated from the app platform of the third-party media, and the content form publicity completely depends on the third-party platform, which inevitably leads to the limitation and restriction of the third-party platform on its content production and profit realization.

Being different from "Ergeng","'Yitiao" has had an independent app platform and has got rid of the limitation of the third-party platform. First of all, for the "Yitiao" micro blog audience, they can only browse the whole short video content on the platform because of the "one short video per day" pattern. Due to the limitations of the micro blog platform, the deep long text content cannot be presented in it. For some text audience users, "Yitiao" will publish the whole video content and text content on the app after its own app platform is launched. The audience who wants to read the text deeply must open the content link of app to read the text completely.

Secondly, for the public account audience, because of the restriction on the number of video uploaded in the WeChat public account, "Yitiao" directly relates the public account content to a "living hall" applet, which is basically the same as that of APP, and only the related small program can be opened by the audience, then the whole video and text content can be seen.

Through the establishment of its own app platform, "Yitiao" almost completely shakes off the content control of the third-party media platform, and integrates multiple media "tributaries" to finally import it into its own app platform, and its app gets the maximum publicity and promotion in this form of media integration. Having got "Yitiao" makes app accumulate a large number of user audiences in a short period of time by getting rid of the shackles to guide the audience with multi-point propaganda by means of media integration.

\subsection{Aesthetics Laboratory: “Immersive” Experience, Online and Offline Interconnection}

The part of the offline experience store, such as freshippo store, Tmall offline experience store, etc., depends on the traditional online e-commerce platform. There are almost two separate individuals between online and offline shopping experience, and there is no strong interactivity.

Unlike the offline experience store of the traditional e-commerce, "Yitiao" opened three offline experience stores in Shanghai in 2018-2019, and connected online and offline experience. "

The "Aesthetics Laboratory" still follows the core of "Yitiao" - life aesthetics, through a free lecture experience in different offline experience stores, combined with online APP promotion. After the launch in October 2018, in the traditional consuming space in the mall, "Yitiao" have created a unique "cultural palace" covering all kinds of life fields. It regularly invites well-known professionals for "face-to-face" communication, and the lectures will be broadcast live on the Weibo platform.

\subsection{Crowdfunding Platform: "Resonance" Crowdfunding to Strengthen the Internal Core Value of Products}

Because the unique content orientation of "Yitiao" strengthens the core value of products, the crowdfunding products can move people's hearts to a greater extent.

Crowdfunding products are usually designed by some civilian designers in combination with life aesthetics to have practical use value of handicrafts, closely around a core of "Yitiao". On the one hand, combined with a strong content production team, crowdfunding products are often combined with in-depth articles. Usually a crowdfunding 
product, combined with an in-depth interview with a product manufacturer, not only starting from the actual value of the product itself, but also focusing on the emotional resonance between the product manufacturer and the audience. This kind of resonance based on the feelings of the manufacturer highlights and deepens the public welfare and voluntariness of crowdfunding, which is more direct to the people's heart and easier to touch the emotional pain of the audience.

On the other hand, the crowdfunding platform of "Yitiao" once again shows the value core of the platform. We can see that crowdfunding products almost cover all fields, such as culture, art, photography, architecture, professionals, food and lifestyle. This is highly consistent with the "Yitiao" short video content. In short video classification, there are photography masters, classic architecture, character stories, interior renovation, social topics, professional spirit, film documentary and other classifications for audience to see. After the audience's basic cognitive training and recognition of "Yitiao" short video content core, it will be easier to establish spiritual connection with the corresponding crowdfunding platform, so as to achieve the ultimate goal of product crowdfunding.

\section{The "Yitiao" App Dilemma of Innovative Business Model}

The cultural industries "are vital to the creation of jobs and the growth of our economy. The creative and media industries worldwide are growing rapidly - we must grasp the opportunities presented". In addition to its innovative "content e-commerce" and "immersive experience", there are also some inevitable gaps. The gaps and dilemmas are mainly reflected in the following parts:

\subsection{Commercialization Is Turned Upside Down, Causing Content to Backfire}

In the past, advertising was monetized only through content, often extremely high-quality short video soft content. In this process, advertising content itself is also a reflection of platform values, and video advertising cannot be separated from content decoration. Sometimes, compared with the daily content of the platform, it will be more prominent and sophisticated, and the audience's feelings from it are selective. Even if it has commercial intention, the content itself will not be completely consumed by commercial monetization, or even go to another direction, which is better and sophisticated, so as to better consolidate the audience group.

Many advertisers of specific brands also tend to adopt the form of "soft advertising" of short video. In 2018, more than half of the advertisements launched by sports functional brands such as "Nike", "Adidas", "Puma" were in the form of "soft advertising" in short video, while the international first-line luxury brands such as "Chanel", "Dior" and "LV" took the form of "soft advertising" of short video as the main form of brand value.

However, a big problem brought about by the e-commerce monetization of "Yitiao" is the content backfire. First of all, unlike advertising cashing, e-commerce is a completely commercial way of cashing. In this process, the audience pays more attention to the practical value of the product itself, rather than the spiritual connotation of the product. In particular, the items sold in a life museum are widely used. In addition to cultural products, it is very difficult for basic life items such as socks, towels, clothes and so on to have spiritual content. If there is a connection, it will have to go back to the original e-commerce model again, which cannot be connected with the innovative "content-based e-commerce".

Secondly, because of this liquidity mode, "Yitiao" recommends selling products through public numbers and so on. Once a day, only a short video of "Yitiao" has been launched. It has gradually become a "video" attached to the chain, the seven selling product recommendation. It looks more like a public product selling recommendation products, rather than a cultural self-media for short video content.

The pure commercialization of putting the cart before the horse makes "Yitiao" backfire on a large number of senior fans. Therefore, how to grasp the balance between commercialization and content-based stuff, and how to retain the audience of fans with content as the core, are still the issues to be explored in the future realization transformation of "Yitiao".

\subsection{The Technology Has Not Reached the Peak, Resulting in Untimely Feedback}

On the one hand, in the APP, because the type of users has limitations, especially the "Yitiao" has clearly targeted the user to the middle class, so in the feedback evaluation of the purchase of products, the number of feedback is very small, it is difficult to generatethe criteria for user reference and evaluation at the time of purchase.

In addition, the combination of the "offline experience store" and the "online aesthetics laboratory" can give consumers an immersive experience and professional aesthetics, but this experience is only based on third-party services and is not proactive. Like many offline experience stores, the best way to focus on experience is to bring in the technology of virtual reality. 
The use of 360-degree panoramic technology to spread the core of the enterprise in China has already achieved great development. In the industry that focuses on content-generating income, this method is mostly used for WeChat moments advertising. The advertisement of the moments promoted by Lincoln Voyager on July 31, 2019 in mainland China, with the "test drive" as the core, 360-degree experience was used to show the advantages of this model, allowing consumers to maximize the 360-degree scientific and aesthetic experience. Tencent's advertising campaign launched in August this year - helping her to go home as soon as possible using 360-degree panoramic view, and made it possible forl the audience to experience tracking clues, resulting in great empathy.

Using 360 panoramic immersive advertising, one advantage lies in the fact that its own app is not subject to third-party constraints. The 360 -wide offline experience hall is launched online, combined with strong content depth to attract consumers to connect online and offline.

In the offline experience hall, we should combine advanced technologies such as virtual reality to strengthen the aesthetic core of the brand and broaden the audience through experience. VR technology combines experience and narrative especially with technical advantages. For short video startup teams that rely on content, the high-quality content is seen and detailed, and the audience's experience is enhanced to truly understand the core value transmitted by the brand.

\section{Conclusion}

After the content matures and deepens, the innovative business model is unique, bringing the new media features of contentization, resonance and interconnection into the commercial e-commerce field. Nowadays, innovation combines the offline cultural output. When almost all short videos are realized in the context of advertising content, the e-commerce realized by "Yitiao" is very distinctive. However, the problems arising from this cannot be ignored. Without the support of strong advanced techniques, the core content is commercialized and countered, and it is impossible to compete with large-scale mature e-commerce, which may affect the vitality of the monetization of content e-commerce. .

Finding the balance between business and culture is not only the core issue of the transformation of short video from the media, but also the problem that the global media industry needs to think about and overcome because of the emergence of different media forms and the development of science and technology in the new media era.

With the short video industry booming, the "Yitiao" provides an innovative way to make the industry think about the new possibilities of entrepreneurial self-media monetization, also exposes the industry limitations of content transformation. Thus how to break through this limitation and how to find a balance point of realization will be a problem that people would discuss eternally.

\section{References}

Atkinson, S., \& Kennedy, H. W. (2018). Extended Reality Ecosystems: Innovations in Creativity and Collaboration in the Theatrical Arts. Refractory: A Journal of Entertainment Media, 30(2018), 10.

Cunningham, S. (2002). From Cultural to Creative Industries: Theory, Industry and Policy Implications. Media International Australia, 102(102), 54-65.

Diego, S. (2009). Internationalization, Design and Global Development. IDGD: International Conference on Internationalization, Design and Global Development, 5623, 19-23.

For, S. J., \& For, S. J. (2003). The Theory of Economic Development. Joseph Alois Schumpeter.

Garnham, N. (2005). From cultural to creative industries. International Journal of Cultural Policy, 11(1), 15-29.

Jenkins, G. M. (1980). The coming of post-industrial society - a venture in social forecasting. Journal of the Operational Research Society, 31(1), 83-84.

Jia, C. (2018). Research on the production and operation of short video "Yitiao". Sichuan Normal University, 84.

Ko, Y. Y., Lin, P. H., \& Lin, R. (2009). A Study of Service Innovation Design in Cultural and Creative Industry. International Conference on Internationalization, Design and Global Development, 5623.

Labour Party. (1997). Create the Future: A Strategy for Cultural Policy, Arts and the Creative Economy. Labour Party, London.

Matheson, B. (2006). A culture of creativity: design education and the creative industries. Journal of Management Development, 25(1), 55-64.

Morag, S., \& Tarek, V. (2017). Cultural Policy, Innovation and the Creative Economy. https://doi.org/10.1057/978-1-349-95112-3

Postman, N. (1985). Amusing Ourselves to Death. ETC: A Review of General Semantics, 13-18. 